\title{
A review of quality procedures in the UK forensic sciences: what can the field of digital forensics learn?
}

\author{
Page, H., Horsman, G., Sarna, A and Foster, J.
}

\begin{abstract}
With a growing reliance on the various forms of forensic science evidence in complex criminal investigations, the measures for ensuring its quality are facing increasing scrutiny. Improvements to quality management systems to ensure both the robust application of scientific principles and the accurate interpretation and reporting of results, have arisen as a consequence of high-profile rebuttals of forensic science evidence combined with process improvements driven by evaluation of current practice. These improvements are crucial to ensure validity of results as well as maintaining the trust of all those involved in the Criminal Justice System. This work first examines the quality management systems utilised for the examination and analysis of fingerprint, body fluid and DNA evidence. It then proceeds to highlight an apparent lack of comparable quality assurance mechanisms within the field of digital forensics, the newest branch of forensic science. Proposals are provided for the improvement of quality assurance for the digital forensics arena, drawing on the experiences of, and more well-established practices within, other forensic disciplines.
\end{abstract}

Keywords: Forensic Science; Digital Forensics; DNA, Fingerprint; Quality Assurance; Peer Review; Codes of Practice and Conduct.

\section{Introduction}

In 2018, law enforcement and associated forensic practitioners are tasked with investigating large volumes of some of the most complex forms of organized crime to date (Skelly, 2018). As a result, arguably almost all serious crime will at some point during an investigation, require input from at least one of the branches of forensic science to assist with evidence collection, analysis and reporting. Often this task involves cross-forensic disciplinary teams working together to analyse multiple evidence types and collaborate in the context of interpreting potential evidence in a robust manner, for presentation in court (Morgan, 2017). The reliance on forms of forensic evidence to support criminal justice processes has arguably never been greater (Ludwig and Fraser, 2014), where in some cases such content may be the sole means of seeking a prosecution. Whilst judicial systems are now reliant on forensic evidence as a means of effectively investigating and policing crime, concurrently, the various disciplines of forensic science find themselves subject to some of the highest levels of scrutiny they have ever encountered (Ireland and Beaumont, 2015). A growing concern that forensic science standards are falling below what might be expected to exist, or in some cases have already fallen, is surfacing in both media (BBC News 2017a, BBC News, 2017b; BBC News, 2016; BBC News, 2015a), Parliamentary (Parliament.tv, 2018) and academic (Collie, 2018) platforms, where miscarriages of justice as a result of ineffective forensic practices have been reported (for example, the case of Colin Pitchfork (BBC News, 2012; BBC News, 2015b)). The following concerns surrounding forensic science standards have been expressed by the UK Forensic Science Regulator, Dr. Gllian Tully.

With the multitude of competing pressures on senior police leaders such as safeguarding, anti-terrorism and serious crime, it is clear that ensuring 
quality has sufficient priority and attention has not yet been consistently achieved. There is a substantially different dynamic in large forensic science providers, whose only business is forensic science provision. Whilst it is understandable that senior police leaders have a wide range of priorities, if quality of forensic science provision is of insufficient priority to enable risks to be managed effectively and quality standards to be achieved, the logical result is that it will become unsustainable for any forensic services to be managed within some police forces (Tully, 2017).

In many criminal justice systems three sub-forensic disciplines are frequently called upon for the purposes of victim/offender identification and event reconstruction, namely digital forensics (DF), fingerprint examination and DNA analysis, with their overarching duty the same; to provide an accurate interpretation of their respective evidence types. One of the difficulties faced by practitioners in the forensic sciences lies with the fact that "expert evidence is a contentious area with a number of high profile cases highlighting unreliable "scientific" expert evidence, leading to appeals and acquittals" (Ireland and Beaumont, 2015 p.1). As both investigation techniques develop and the speed of new knowledge discovery increases, the discipline is better placed to scrutinise this previous work. This has been witnessed in a range of investigatory areas and noted by Smit et al., (2017). In addition, forensic science services are operating on a smaller budget with stricter time constraints and higher workloads (Science and Technology Committee, 2014). As a result, greater emphasis is placed upon the appropriate use of new technologies to produce value-formoney outcomes, whilst remaining within the constraints laid out in those systems designed to ensure the quality management and delivery of reliable investigatory findings (Kleuskens, 2015).

Given the increased external scrutiny placed on all publicly funded services, it is the production of 'reliable findings' in cases which is key. In some forensic arenas, the quality management systems to ensure the production of 'reliable findings' are well-developed. They have evolved over an extended period of time and have been exposed to both external and internal scrutiny. There are other, less mature disciplines, such as digital forensic science, where this is not the case. Limitations in quality management systems in digital forensics (DF) has been alluded to in oral evidence presented to the Justice Committee in May 2018 (Justice Committee, 2018).

The contribution of this article is twofold; first, the quality management systems in place for fingerprint examination and body fluid and DNA analysis are examined in detail. Second, an apparent 'lack of' quality management in DF is highlighted and its impact on criminal investigations and evidential reliability is assessed. Recommendations for DF are offered drawing attentions to lessons which can be learned from other forensic sciences as they seek to ensure the quality of their work.

\section{Scrutiny: Pivotal Cases Driving Process Development}

Whilst all sub-disciplines have faced scrutiny, arguably, DF has escaped the large scale public interrogation and inquests faced by fingerprint examination and DNA analysis. In comparison to fingerprint examination (in use for over 100 years (Walvisch, 2017)) and DNA analysis (first utilised in 1988) (White and Greenwood, 1988), DF is a relative 'newcomer' to the forensic scene. A lack of scrutiny may impose a false sense of security with regards to 
the reliability of evidence provided by this discipline, particularly as it is operating under arguably less rigorously defined standards, practitioner governance and evidence validation procedures. In comparison, fingerprint, body fluid and DNA evidence, and their associated procedural practices, have been questioned in a number of cases including the Marchioness disaster (Clarke, 2001), the 'Madrid Bombings' (Cohen, 2012) and the Damilola Taylor case (Rawley and Caddy, 2007).

\subsection{The Caddy Review}

In 2007, the analysis of Low Copy Number (LCN) DNA was called into question after Sean Hoey was cleared of the murder of the 29 people who died in the Omagh bombing. One component of the case was the identification of DNA on the bomb timers, linked to the attack, as originating from Mr. Hoey. However, this was challenged by defence experts, particularly in respect to the reliability of the laboratory method, and the judgement laid down in the case included commentary relating to the preservation of evidence integrity from the moment of seizure (NICC 49, 2007). The Caddy Review, reporting in 2008, concluded that completion of Low Template DNA (LTDNA) analysis of LCN was "sound" and the methods used were validated consistently against standard scientific principles. However, recommendations were made in relation to the interpretation of DNA profiles produced using this method of analysis, introduction of standardised quantification of DNA before profiling, appropriate training, use of DNA-free consumables, monitoring of accreditation and validation documentation and research. These recommendations have been incorporated within the Codes of Practice and Conduct (for forensic science providers and practitioners in the Criminal Justice System) (CoPC) (2017a).

\subsection{The Fingerprint Inquiry}

The Fingerprint Inquiry was launched in March 2008 to publically examine the identification and verification fingerprint evidence provided in the case of HM Advocate v McKie in 1999. Ms Shirley McKie was prosecuted for perjury at the trial of a murder investigation in which her fingerprint was identified within the house of the victim. At the trial, Ms McKie denied being present in the house beyond the porch. Ms McKie was found not guilty and a subsequent enquiry found that the fingerprint had been misidentified. The outcome from this enquiry, the successful appeal of the original murder conviction, the settling of Ms McKie's civil claim against Scottish Ministers and the outcomes from a parliamentary inquiry which identified "widely divergent professional opinions" (Justice 1 Committee Report, 2007), all culminated in the call for a full inquiry in to the McKie case.

The Fingerprint Inquiry concluded that there was misidentification of fingerprints in relation to the original murder case but that there was "no reason to suggest that fingerprint comparison is an inherently unreliable form of identification" (Section 34.7, The Fingerprint Inquiry, 2011). Part 8 of the Report summarises the key findings and 86 recommendations of the inquiry which span all aspects of fingerprint evidence, examination, recording of notes, training, accreditation and research. In a similar manner to those recommendations from the Caddy Review, these have been incorporated into the processes and procedures currently employed within the UK.

\subsection{What about DF?}

To date, DF has remained relatively unchallenged with regards to high-profile reviews of its capacity to provide reliable evidence, instead attracting recent criticism around procedures 
for the prompt disclosure of digital evidence (see the recent case of Liam Allan (Bowcott, 2018)). Yet, its effective application is beginning to attract some media attention and scrutiny (see Smith, 2018) where Murphy (2007) warns that such modern techniques should not be wholesale embraced without hesitation. Perhaps one of the only main cases to attract attention was that of Operation Ore, regarding the ineffective investigation of Landslide Productions Inc. in 1999, in relation to indecent images of children. Although the predominant evidence types were digital in nature, issues lay with the effective investigation of information as opposed to interpretation and presentation of specific digital evidence types. Here apparent failure to verify system functionality in relation to credit card transactions, and their associated access to material, meant that many individuals were considered to have breached the law, having not actually done so. Although the fall out of Operation Ore could be considered significant with appeal cases considered some 10 years post initial investigation, it cannot be considered to have shaped DF standards. Minor examples include that of the misinterpretation of results from the CacheBack software in the US (Wilson, 2011).

The field does not maintain the developmental milestones witnessed in other forensic sciences, brought about through public scrutiny, yet this should not be a measure of reliability, instead caution is argued. Only recently are we witnessing the refuting of forensic science principles in traditional forensic science disciplines once considered reliable, which have been in operation for many years. The continuous development and testing of techniques, afforded by time, has now meant an increased rigour in procedures. As DF has not yet 'stood this test of time', arguably it has not yet been opened to such scrutiny. It may tentatively be assumed that such issues might exist but are yet to be discovered. As previously identified, in order to circumvent issues of reliability, validated, robust quality management systems should be in place for all branches of forensic science including DF.

\section{General Standards for Quality Assurance}

Since 2011, the Forensic Science Regulator has published Codes of Practice and Conduct (for forensic science providers and practitioners in the Criminal Justice System) (CoPC) (2017a) with the aim to establish/identify the quality level required within forensic investigations. The subsequent/concurrent accreditation mechanisms by the United Kingdom Accreditation Service (UKAS $\mathbb{R}$ ) are a $\mathrm{m}$ eans to dete $\mathrm{m}$ ine whether these standards are $\mathrm{m}$ et These CoPC align with BS/EN ISO/IEC 17025 standard (for testing and calibration laboratories as interpreted by ILAC G19:08/2014), hereafter referred to simply as ISO 17025 (BSI Standards Limited, 2018), providing context in relation to the UK environment. The latest complete set of CoPC, published in 2017, contains additional information in relation to digital forensic evidence analysis. The CoPC focus on the assurance of quality, via procedures for aspects such as document control, validation testing, method validation and training and competency of staff (recommended to be linked to the National Occupational Standards). However, the standards do not specify how investigations should be undertaken - the methods used can be provider specific providing they are appropriately validated according to these standards. One aim of the ISO standards is to reduce the likelihood of both practitioner and instrument error (Christensen, et al, 2013) and any subsequent appeals based on forensic evidence. It is argued (National Academy of Sciences, 2009) that such state oversight for the regulation of forensic science providers is critical to ensure that standards are consistently applied, thus aiming to prevent such problems arising. 
Quality assurance (QA) is defined within ISO 9000:2015 Fundamentals and vocabulary, Section 3.3.6 (2015a) as "part of quality management focused on providing confidence that quality requirements will be fulfilled". In essence, it is a way to prevent errors occurring during the production of a product or service. QA ensures that standards and procedures are established within a working environment and reviews these so that improvements can be made with respect to how a process is performed. Quality control is similarly defined (in section 3.3.7) as "part of quality management focused on fulfilling quality requirements" and is a way of ensuring that the results obtained are what were expected, so how 'correct' the product is.

Within the CoPC, it is stated that technical records should:

"be clear and comprehensive, and expressed in such a manner and in sufficient detail that another practitioner in the same field, and in the absence of the original practitioner, can follow the nature of the work undertaken, any interpretations/opinions made, and the inferences drawn from the work." (Forensic Science Regulator, 2017a).

The contemporaneous recording of the examination of exhibits in this way permits the subsequent checking and review of critical findings by a competent individual ${ }^{1}$. According to Ballantyne, et al (2017), this takes two primary formats within evidence presentation: 1) peer review and 2) verification. Where peer review involves the checking of reports and statements for accuracy and appropriate interpretation, verification involves the repetition of the analysis stage by a second, independent examiner. Different disciplines within forensic science employ either peer review or verification. Lessons can, therefore, be learnt from differing disciplines' peer review/verification processes which could be more widely applied, particularly within the field of digital forensics. It is the aim of this work to identify what processes are employed and establish some 'best practice' guidelines for implementation.

\subsection{What do other sciences do?}

It is important when considering the practices of one discipline, particularly one considered relatively 'new' to the forensic arena such as digital forensics, to review the current practices of more long-standing and established disciplines. This therefore allows one to learn from those established disciplines and consider their processes in-line with the newer discipline, in order to ascertain the relevance and applicability (or not) of such practices for the development of standard ways of working. That is not to say that those established disciplines are perfect in their undertakings, more that it is sensible to not require the reinvention of the wheel for each new discipline. In this instance, the disciplines of fingerprint, body fluid and DNA examination are used for comparative purposes.

\subsection{Fingerprint examination}

The process of fingerprint identification relies on the skills and expertise of fingerprint examiners and is conducted via the ACE-V methodology. Usually, the unknown fingerprint (also known as the latent print or fingermark) is compared against a known fingerprint example (tenprint) via: Analysis, Comparison, Evaluation and Verification (Forensic Science Regulator, 2017b). The ACE-V method combines qualitative and quantitative analysis of

${ }^{1}$ A competent individual is defined in ISO 9000:2015 Fundamentals and vocabulary (section 2.2.5.3) as someone who can "understand and apply the skills, training, education and experience needed to perform their roles and responsibilities" (2015a). 
friction ridge detail. According to the Scientific Working Group on Friction Ridge Analysis, Study and Technology (SWGFAST), the ACE-V stages can be described as: 1. Analysis assessment of the impression to determine value, possible anatomical origin and orientation; 2. Comparison - side-by-side observation to determine similarity or dissimilarity; 3 . Evaluation - determination of sufficient information to exclude or individualise, or whether there is an inconclusive conclusion drawn; and 4. Verification - independent application of the ACE process to support/refute the original conclusion (SWGFAST, 2013, Forensic Science Regulator, 2017b).

When undertaking a fingerprint examination, historically there was little consistency in practice and quantity of contemporaneous information recorded at the time of examination, due to time constraints in operational working. Basic information such as the digit, and the demographic details of the identified person were recorded if the comparison was considered an identification. However, as a consequence of the introduction of the CoPC there is now a direct stipulation in section 15.2.2 for contemporaneous technical records which are sufficient in detail to permit "another practitioner in the same field, and in the absence of the original practitioner, can follow the nature of the work undertaken, any interpretations/opinions made, and the inferences drawn from the work." Compliance with this aspect of the CoPC is variable at this stage, and the manner in which information and type of information that is recorded, also differs.

Within the UK there is variability and inconsistent practice, with respect to agency policy, in relation to the verification stage of identifications/non-idents/elimination idents. The Codes of Practice and Conduct: Fingerprint Comparison (2017), state that verification is completed by a second competent examiner in an independent manner to verify the original outcome. However, it is not stated as to whether this practice should be applied consistently for all outcomes of the original ACE examination, for example whether Verification should be consistently applied for all identifications, non-idents and exclusions. Customarily, bureaus would not normally complete a Verification of a non-ident (although some bureaus would complete Verification on all outcomes), and practice in relation to Verification of an elimination Ident varies.

Depending on bureau practices, the Verification stages are a single or double check of the outcome from the Evaluation stage. Normally, if a 'non-expert'2 completes the original ACE, the Verification stage would be undertaken twice by two different expert examiners, the third check usually completed by a senior member of staff (Senior Examiner/Team Leader/Head of Bureau). A two-stage Verification process would be employed if the initial Verification was undertaken by an expert, and the check was subsequently completed by a (senior) expert. Ulery, et al (2011), report that, although false positive and false negative idents are made, independent examination, equivalent to the process of blind verification, detected all false positives and "the majority" of the false negative results. Thus, there is a certain degree of assurance that this verification stage can limit errors in identification. However, converse to this, concerns could be raised with respect to the final verification, and ultimately the final decision, lying with the 'senior expert' within the bureau, as it is likely that this individual

\footnotetext{
${ }^{2}$ Non-expert in this instance is defined as a fingerprint officer (or similar) who is deemed competent in examination but who has not yet qualified to advanced level.
} 
would complete less comparison work than anyone else due to other work-related demands on their time.

In addition, the assurance of reproducibility and associated quality procedures, and a reduction in false negatives (Langenburg, et al, 2009) can be determined through the use of blind verification. Blind verification can be deployed in situations such as complex examinations (Recommendation 9, The Fingerprint Inquiry, 2011), when there is conflict between examiners during the Verification stage, or where there are few features, in addition to the random dip-sample selection of cases for quality control purposes.

Drivers towards increasing the efficiency and cost effective nature of case examinations have meant that there are now additional timescale pressures. One of the outcomes of this was the revision of case management and the introduction of streamlined forensic reporting (SFR) in 2011 bringing significant benefits to all involved.

SFR is a two stage process which can be particularly effective in increasing the rate of guilty pleas, avoiding unnecessary forensic and prosecution work, leading to a reduction in costs and delays in the criminal justice system. A short report (SFR1) is initially produced which identifies and summarises the key forensic evidence in the case, and is suitable for charging purposes and initial court hearings. A second report (SFR2) is produced if further clarification is needed or if there is dispute by the defence over conclusions drawn within the SFR1. The SFR2 only highlights those areas requiring clarification or which form part of the disagreement.

The aim of the SFR process is to achieve early agreement with the defence on forensic issues whilst decreasing the need to produce a full forensic report of the findings. If the agreement with the defence cannot be achieved in the first instance, the contested issues are identified and explained so the court understands more about the possible issues arising from the fingerprint evidence in the case. It also helps reporting experts to be more prepared for the possible lines of questioning from both the defence and prosecution advocates. This was also discussed by the Right Honourable Lord Justice Gross, who states that:

"SFR can deliver significant benefits to the courts, prosecution and defence. Court time is saved. Unnecessary forensic work is avoided. Unnecessary prosecution work is avoided. The defence are better able to focus on the real issues and appropriately advise their clients. I urge the judiciary and all parties to ensure that it is used appropriately and in accordance with the Rules." (CPS, 2017)

For fingerprint evidence, the reporting of results within court previously was the responsibility of individuals who were registered on the National Register of Police and Government Department Fingerprint Experts, governed by the Association of Chief Police Officers (ACPO), at the College of Policing (CoP). In 2015, ACPO was disestablished and, in its place, the National Police Chiefs' Council (NPCC) was formed (NPCC, 2018). However, the register was never renamed and remained at the CoP, operating under the old ACPO guidance policies and maintained by the CoP.

The purpose of the Register was to ensure standards of competency within the profession and validate the expertise of those fingerprint practitioners reporting and presenting 
evidence in court. On-going registration was dependent upon the demonstration of continued practical competence. However, the court has accepted fingerprint evidence being presented by those not appearing on the register, particularly independent defence experts. Despite this, in $R V$ Smith [2011] EWCA Crim 1296 one 'defence expert's' qualifications were called into question and deemed not acceptable or recognised in the UK. The decision was taken by the judge not to call them to provide evidence in the case

In order to become a 'Registered Fingerprint Expert', one was required to successfully complete the UK National Fingerprint Learning Programme (NFLP) at the College of Policing (or equivalent programme delivered by the Metropolitan Police). There was no opportunity outside of the UK to become a UK recognised fingerprint expert. However, since the decision to cease delivery of forensic training by the CoP the issue of training to become a fingerprint expert is now under scrutiny. Currently, the CoP are in the process of writing the learning standards for a revised programme, in order for it to be licensed to forces for local delivery. What a localised delivery approach might look like in terms of quality assurance of who and where the training will be delivered is still in discussion. Deliberations are currently underway within and between both forces and universities as the NFLP (and Metropolitan Police equivalent foundation degree) was recognised and accredited by Teesside University (Teesside University, 2008).

By October 2018, all fingerprint bureaus are required to be accredited under ISO 17025. One approach in relation to the training and competency checking of Fingerprint Experts would be to link this to the ISO 17025 accreditation. Thus, those individuals at an appropriate level who are fully trained and working competently within the bureau would then be considered an expert. This would then remove the need for a register of individual experts, but would place the assurance of training standards and quality compliance of competency solely within the remit of the bureau.

The fingerprint community are also examining the current role profile of a typical 'Fingerprint Expert'. This new approach will enable a bureau to have both non-reporting fingerprint practitioners and reporting fingerprint practitioners. They will all be qualified to advanced level in fingerprint identification, however, only those reporting officers will undertake training in preparation and presentation of evidence. This is a slight shift from the historical career pathway of a fingerprint expert. At present, there are some bureaus operating in this way with only a limited amount of reporting officers.

\subsection{Body fluids and DNA Analysis}

The examination and recovery of body fluids is intrinsically linked to that of DNA and its subsequent analysis. The examination for body fluids relies on a range of presumptive and confirmatory tests primarily to identify blood, semen and saliva. Once biological or cellular material, such as dandruff, is identified/recovered, it is followed by the analysis of that biological material to obtain a DNA profile. The processes for body fluid and DNA examination and analysis are outlined within ISO 17025 standards and the CoPC.

The formal date for achieving accreditation to ISO 17025 for "Processing recovered biological samples/material to obtain a DNA profile" and for "Blood pattern analysis" was April 2012. The ISO 17025 accreditation date for "Visual screening, examination and/or sampling for biological material" was October 2013. Additionally, accreditation scope was 
widened to include the CoPC for each of these areas with an associated deadline of October 2017. However, accreditation to the CoPC is variable across the industry. This is due to the voluntary nature of compliance at this time because of the lack of statutory power for the Forensic Science Regulator. This means that a number of different, but well-established quality assurance (QA) measures are in place, and all examinations of body fluids and any subsequent DNA analysis must be undertaken according to ISO 17025 and can conform to the CoPC.

\subsubsection{Critical Findings}

As soon as human interaction is introduced in to a process, there is the possibility of humanrelated error. As a consequence, according to ISO 9001 Section 8.5.1.g (BSI Standards Limited, 2015b), actions to prevent human error should occur. In this instance, all critical findings are quality assured/checked by a competent individual authorised to carry out such checks. Although practice varies within establishments, to be permitted to complete authorisation checks, the person must be both fully competent in that discipline and fully trained to carry out such checks. Outcomes, and the authoriser responsible, are recorded, irrespective of whether the critical finding was agreed or not.

The Codes of Practice and Conduct (Forensic Science Regulator, 2017a) define 'critical findings' in subsection 15.3.2, footnote 43 , as:

"observations or results that: have a significant impact on the conclusion reached, the interpretation, or an opinion provided; cannot be repeated or checked in the absence of the exhibit or sample; and/or could be interpreted differently."

For example, a 'critical finding' during presumptive testing for the presence of blood pertains to: (a) an ambiguous presumptive test result, for example, a slow developing test reaction; (b) a positive presumptive test result on a stain that does not have the appearance of blood; or (c) a negative presumptive test result on a stain that has the appearance of blood.

\subsubsection{Preparing for Examination}

Although there are differences in practices, generally a forensic strategy is employed to maximize the potential recovery of evidence according to the National Occupational Standard CN 702: Determine the forensic examinations to be undertaken (Skills for Justice, 2013). Simple examinations, such as cellular recovery from basic/straightforward items where the likelihood of any other body fluid is very low, usually follow processes defined in procedures held within a Quality Management System (QMS). For serious or complex examinations, including major crimes, where interpretation of body fluids is required (such as blood pattern analysis) or if there are multiple scenes/suspects in a case, any forensic strategy has to be quality assured before the start of the examination. This peer review process, by a second competent examiner, ensures that the correct techniques are performed in the correct order in order to maximise potential evidential recovery.

Batches of critical consumables should be QC tested and the records held. This may include minitapes, sample tubes and swabs. The equipment that can have an impact on the outcome of the results requires calibration prior to introduction to the laboratory and periodically thereafter (BSI Standards Limited, 2018, ISO 17025: 2017, Section 6.4.6). Use of appropriate QC standards, and regular monitoring of test outcomes with those standards, 
is a part of routine equipment testing. Failure of the standards to behave as expected in a test might disqualify test results, thus maintaining suitable standards is imperative. Laboratories completing the examination of body fluids and DNA should have a documented program for conducting calibration of equipment such as thermometers, weighing scales and pipettes according to ISO 17025: 2017 (BSI Standards Limited, 2018).

When reagents are made in house, they are QC tested before they can be used in the laboratory with known positive and/or negative control samples. In addition, critical reagents ${ }^{3}$ also require a quality control check for day-to-day usage. The results of this QC check should be recorded in the case notes, including the reagent batch number, and there should also be traceability of the expiry dates of reagents using the examination notes. Regular monitoring of reagents and equipment is part of any standard QA program and it can help to recognise potentially emerging issues.

\subsubsection{Blood Examination}

Initial examination for blood is carried out via a visual inspection of an item, followed by a presumptive test. Although presumptive tests for blood are very useful, these tests are not confirmatory and false positive reactions can occur. If the obtained reaction is unexpected, a critical finding check by another competent practitioner is necessary as outlined previously in section 3.3.1. In addition, in instances where direct presumptive tests are completed which are not repeatable, for example removing a fibre from the item and testing it for the presence of blood, a 'critical findings' check must be performed.

Depending on the item, alleged case circumstances and expectations, a second examination, by a competent examiner, may be undertaken after the initial blood search to confirm the result. For example dark items, footwear and weapons, such as knives, are difficult to search and thus it is more likely that blood staining might be missed. However, within the UK there is variability with respect to organisational procedures in relation to the second check of items subjected to blood search. The CoPC (2017a), state that the forensic unit shall have a procedure for carrying out checks on critical findings (Section 15.3.2). What is not stated is whether this practice should be applied consistently for all blood searches or only selected ones. As a consequence, the approach and frequency of second checks varies between laboratories and is founded on a risk-based approach.

Where forces have only a single level of hierarchy within their examiner teams (i.e. all examiners are of the same level, e.g. Forensic Laboratory Officer), positive blood search and recovery examinations are not second checked in terms of being re-examined, they are simply subjected to a case file peer review. In comparison, all blood search and recovery examinations which result in a negative finding are subjected to a second examination (a verification in terms of FP evidence examination) by a competent member of staff. Only if the two examiners are in agreement, will a 'no blood found' result be reported. Even at this stage, the completed case file is then subjected to a case file peer review, in line with section 15.3.4 of the CoPC (2017a).

\footnotetext{
${ }^{3}$ Critical reagents include those used in the presumptive testing for the presence of body fluids.
} 
It is established good practice that all second check blood search examinations are collated to determine any procedural trends, such as blood missed on certain item types, thus providing an evidence-base to inform procedural change. If there is a hierarchy of staff within an examiner team, with Forensic Examiners (FE) and more senior Reporting Scientists (RS), or similar, the approach to checking varies. If a FE completes the initial blood search and recovery, then a RS may have to check all the items. However, if a RS completes the examination, another RS may check only those items found positive or, if all items tested were negative, at least one of these items would be checked. In addition, the RS may decide which items require a second check depending on the case circumstances.

A repeat blood search is carried out when blood pattern analysis is required because any missed bloodstains may have an effect on the interpretation of the pattern. A number of providers follow the blind peer review process as outlined in Osborne and Taylor (2018). Thus meaning that before any stain is sampled, an independent analysis of evidence is carried out, but in the absence of any contextual information or the original examiner's notes or interpretation/s. This mechanism aims to QC the process of BPA, with minimal impact on overall work-flow.

\subsubsection{Semen and Saliva Examination}

The presumptive test most routinely used to screen for the presence of semen, is the acid phosphatase (AP) test. The AP test is to determine the presence of an enzyme found in uniquely high levels in seminal fluid (Kind, 1957). As it is a critical reagent, the AP test should be QC checked prior to every use against Quality Control Materials (QCMs) according to ISO Guide 80 (BSI Standards Limited, 2014). After usage for screening an item, the AP test reaction outcome is reviewed by a second competent practitioner and/or photographed. The examiner will contemporaneously document details of any reactions which allows the RS to be able to assess the information and form their interpretation/s. This will also allow the RS to make a final decision with respect to submission of samples of DNA profiling. The same process is undertaken for the examination of saliva using the Phadebas $\mathbb{R}$ test, $w$ h ich presum ptive ly identifies the presence of eamylase in saliva.

During semen and saliva examinations, it may be necessary to recover multiple samples from an exhibit and transfer these in to sample tubes. This process of transfer is directly observed by another competent practitioner and checked to ensure that samples are not cross-contaminated or transferred incorrectly.

After a positive AP reaction for semen, a microscope slide is produced from the positive sample to allow for the identification of spermatozoa and epithelial cells. The quantity of spermatozoa and/or epithelial cells can be significant for interpretation, therefore a review of the slide should be carried out to QA the original findings. The reagents used to enable visualisation of spermatozoa and epithelial cells should be QC checked when a new batch is created. In the same manner, the test reagents for the Florence lodine test (which tests for the presence of Choline, found in seminal fluid) should be QC checked prior to usage.

\subsubsection{DNA}

During the different stages of DNA profiling (extraction, quantification, polymerase chain reaction (PCR) and capillary electrophoresis), samples are continually transferred into new sample tubes/wells. As previously identified in section 3.3.4, this transfer process is 
witnessed by another competent practitioner to ensure that samples are transferred correctly. The process can be carried out using a suitable electronic witnessing system if available. The correct order of tubes is monitored through independent checking and verification by another practitioner and any discrepancies found are rectified and recorded.

\subsubsection{DNA Quality Control}

To ensure the QC of results, known positive and negative control samples are included with every test for all four of the DNA stages. Negative controls are utilised to provide an indication as to whether there is contamination present in the batch of samples and the positive control is utilised to indicate validity of the process (Forensic Science Regulator, 2016, Section 1.1.7.b.v.). The quantification stage controls can be checked immediately after the completion of the process, but the controls for the remaining stages can only be checked once the final DNA profile is obtained.

\subsubsection{Interpretation of Results and Reporting}

Analysis of a DNA profile is completed independently by two analysts. Prior to examination of casework samples, all controls are checked as, if a failure occurs, stage/s of the DNA profiling process may require repetition. The quality and clarity of casework DNA profiles are analysed to determine whether they meet expected standards in relation to the amount of DNA present in the sample. If unexpected results are obtained, aspects of the DNA profiling process may have to be repeated. This can occur when the standard of a profile is not as high as expected considering the amount of DNA present in the sample, or if a very strong and clear DNA profile is obtained when only a minute amount of DNA is present.

A senior analyst, designated a Final Genotyper (FG), then scrutinises the two analyses before determining the final decision. This includes determining whether the profile requires additional analysis in order to improve it. All suitable DNA profiles are reviewed against an internal elimination database (which includes DNA profiles of staff and any external individuals whose profile is retained) to ensure that no contamination from these individuals has occurred (Forensic Science Regulator 2016, Section 1.1.7.b.i). If a case does not require complex interpretation, the report, including the DNA results for that case, is created by an analyst. The report is peer reviewed by another analyst before it is released to the customer.

Complex cases and complex DNA profiles, with DNA from more than one individual, are interpreted by specifically trained Reporting Scientists. A RS interprets the DNA profiles obtained within the context of the case and produces the final forensic report. A second RS carries out a blind peer-review of the results obtained, where the review is conducted in the absence of the original examiner's interpretation/s. Once this is completed, the second RS performs an administrative check of the final report.

\section{So what about DF?}

Whilst controversial, it is necessary to state that there are few formalised and enforceable peer-review and quality assurance procedures enforced in DF investigations. Typical DF investigations involve the identification, acquisition, examination, interpretation and reporting of digital data (Köhn et al., 2006; Selamat, 2008; Agarwal et al., 2011). In some cases, a DF practitioner may be responsible for all stages, where oversight and evaluation of the work carried out at each point may be minimal. There are currently no governing standards 
depicting what is considered an acceptable peer review of work in DF, or to what degree peer review should take place with regards to any discovered digital evidence. As a result, unlike the quality management systems in place in other branches of forensic science, DF organisations largely self-govern and determine their own levels of acceptable review internally. Those quality systems which exist in DF often target organisations at a laboratory level, not the individual or their outputs and as a result, it is difficult to ascertain the quality and reliability of investigatory work. Such an issue is deep-rooted in DF and recently stated by Sommer (2018. p116):

'We provide evidence that almost by definition a non-specialist audience cannot evaluate for themselves, so that trust is of the essence' (Sommer, 2018 p116).

The effectiveness of any quality management systems (QMS) in DF is impacted by the organisation which are implementing them, and their overarching constraints. Differences between private and public sector DF analysis can be stark and often revolve around resources and profitability. Therefore, QMS may not be considered a mandatory burden which must be addressed, but one which can be manipulated into a form which fits within organisational budgets and resources. In doing so, the effectiveness of QMS may be compromised, where their implementation may be seem as a hoop to jump through in order to acquire tendered work, as opposed to an overarching goal of improving and maintaining quality work. Whilst it could be argued that underlying motivations do not matter as long as standards are met, it should be considered that the implementation of a QMS designed to show adherence on face value is not the same as the whole-hearted adoption and embedding of quality management principles for the long term production of consistently high quality work. In addition, the time given to carry out a DF investigation can differ widely, where private sector practitioners may be costed by their organisation at a fixed mark-up, giving a finite amount of time to complete case work, regardless of factors which may hamper the reality of doing so. In such cases, it would be difficult to argue that quality is not compromised. Whilst costs are often considered a variable that can influence company operation, the cost of litigation triggered by erroneous investigation should be a concern and one which is only likely to increase given the dependency on digital evidence types.

Quality management processes can typically be targeted at three areas: the laboratory, the practitioner and the product. The laboratory accreditation in DF is largely preoccupied with the achievement of the ISO 17025 standard which was announced by the forensic science regulator as being mandatory by October 2017. Whilst this has attracted criticism (see Sommer, 2018) an indeed, issues do exist, with arguments surrounding applicability and the cost of achievement and maintenance often cited, it should be considered a positive step towards the implementation of a standardised regulation for DF organisations, something which is arguably in need. Whilst some may argue that ISO 17025 is the incorrect vehicle for achieving the regulation of standards in DF, it is currently the only viable option in existence for attempting to achieve a measure of quality control.

In the context of achieving and maintaining quality standards, DF suffers from a number of governance issues. The discipline has consistently debated the omission of regulatory standards and entry requirements to be able to practice within this area. Some 15 years on from the raising of initial concerns, DF remains a field where practicing 'professionals' require no formal qualification or certification (Sommer, 2018). As a result, it has been 
referred to as the 'Wild West', a discipline driven and governed by practitioners without oversight of a dedicated formalised regulating body (Sloan, 2015). Without repeating existing concerns (see Meyers and Rogers (2004); Vincze (2016) for regulatory concerns), a lack of regulation can only draw into question the presence of competence in practitioners currently employed in DF and by association, the work they undertake. This is suggested by Pollitt et al., (2017) who state that a "lack of competence can result in errors in forensic results, including overlooked and misinterpreted traces". Whilst no doubt, there are those practitioners with vast experience who are competent to carry out DF investigation, yet we have no formalised ways of measuring this competency beyond arbitrary methods such 'years in industry'. Despite consistent calls for the greater regulation of individuals seeking to enter the discipline of DF and those already practicing, the field is no closer to imposing regulatory requirements on individuals.

Perhaps a concern now lies with the prominence and popularity of digital forms of forensic science resulting in an increase in graduates practicing in this area. Those entering this industry with an undergraduate qualification seek to 'sure-up' the standard of those entering this discipline, but it does not solve all of the issues. Undergraduate DF curricula are themselves unregulated leading to varying standards of coverage of core and specialist areas of DF. Therefore, it is likely that no two graduates will possess the same knowledge set, where any gaps in coverage may be crucial in terms of quality assurance of investigation work.

In comparison to other forensic sciences, DF maintains no formalised entry requirements for wannabe practitioners to enter the field and as a result, the entry gate is manned by those organisations seeking to employ individuals. The role profile and job requirements are wholly defined by those entities, no external prerequisites are required (adherence to a governing body), a candidate must simply meet the criteria defined by the organisation. Advertised roles will often be defined based on a level of predetermined acceptable risk by an organisation where issues caused later may tarnish an organisation's reputation and possibly their sustainable business model. This situation would suggest that the industry will adopt the burden of self-regulating entry requirements, taking only those candidates deemed suitably qualified and knowledgeable. However, in reality, employment is likely to also be driven by business needs including available workload and affordability of additional staff and, given that many private sector firms rely on the volatile nature of tendered contracts, high-turnover of staff may be witnessed. This may well lead to the inevitable employment of individuals who may have less than adequate experience and qualifications who are seeking to gain industry experience and willing to accept the potential short-termism of the role. In such a case, the burden is placed upon the organisation to upskill the individual and vet their work, however this is unlikely to be completed to an effective standard given the amount of time and resources needed to achieve this. Instead, an individual may simply receive training to complete a set of repeatable investigative tasks in order to cope with current productivity demands, without fully understanding any underlying principles, known as surface learning (Higher Education Academy, 2018). This approach undermines attempts to improve and maintain quality.

At a product level, in the confines of DF, this is the investigation undertaken and subsequent interpretation of results, typically captured in a statement/report format. The results of which must be reliable in order for related sound legal decision making based on report contents to 
be made. The problem in DF is that, unlike other forms of forensic science, a practitioner may not have their work effectively peer reviewed or verified before it is given to a client. Peer review of work is a challenge in DF. Often evidence types vary (albeit under the umbrella of digital), where many operating system or application artefacts may be important to an offence. A single entity 'peer-review' (a review of a single entity/evidence type) offers a cost effective measure to those sciences which deal such evidence, however DF is different. In any one case, multiple types of digital data may be exhibited (for example, Internet artefacts, specific software application logs etc). In each case, the time and effort required to check or fully verify each and all of these results grows. Further, the standards of the reviewer may also be brought into question given the issues previously stated in this work.

To start the question must be asked; 'what does an effective peer review look like?', and there are many variables to consider. Assuming that the peer reviewer is competent to carry out this task (see Section 3, footnote 1), to align with other forensic disciplines, peer review must be a process which checks and verifies the work that the original practitioner has undertaken as being correct. Regardless how qualified a practitioner is, everyone is susceptible to human error and the role of peer review is to prevent issues from remaining undetected. Peer review must also be practical and fit within the demands of both an organisation and the overarching criminal justice process. Peer review needs to support those involved to ensure robust evidence whilst also not hampering processes involved with respect to effective and timely delivery of justice. Whilst there are competing interests at foot, nothing should compromise the presentation of reliable evidence.

The extent of a peer review in DF could be considered hierarchical, with the following five levels.

Re-examination: A complete re-examination of the original case and cross-referencing of results. The process is completed blind, followed by a comparative review and discussion of findings between the primary and reviewer's investigation.

Verification review: A full validation review is in essence a second examination carried out across the principal examiners data set only, not all of the acquired digital data, with prior knowledge of the primary practitioners' results set. It allows steps to be re-traced, hypotheses to be confirmed and findings to be validated. In doing so, the chance of misinterpretation is arguably lessened.

Conceptual peer review: This type of review focuses on an individual's description of evidence artefact types, ensuring that they have documented the true interpretation of what they do. Here, there is no check on quantification of evidence, the collection process is assumed to be correct. Instead, it is a check and evaluation of whether the practitioner has conveyed the true understanding of specific evidence types.

Sense review: A check of work to ensure it makes sense as a piece of deliverable evidence. Technically, no checks of evidence of evaluation take place.

Proof check: Here, the work is proofread purely for grammatical and spelling issues. 
Arguably, entry onto the above scale of peer review will be dependent on available resources, knowledge and time. If we consider that practitioners may be costed on fixed 'per-hour' rates, re-examination and verification reviews are unlikely to fit within the organisations costing model. In such cases, the two most effective measures of peer review are likely to be omitted from the examination process, where lesser methods are likely opted for. It is also necessary to consider that in some smaller DF organisations, internal specialisms may exist where it may not actually be possible for two equally qualified individuals to check each other's work. As a result, experts may simply not have access to other practitioners who have an understanding of the niche work they may complete. In such cases, this work may enter the Criminal Justice System based on a singular view.

Non-peer reviewed work increases the chance of misinterpretation by the client. In the context of law enforcement, the practitioner:Officer in Charge (OIC) relationship is key and misinterpretation or issues arising here can have significant impact on an investigation. This has recently been alluded to by Collie (2018) who in relation to OICs, states the following.

"S/he will look at the outputs and decide whether they provide useful evidence. Even where the phone is sent off to an external forensic lab, as happens in other parts of the UK, the procedure will be the same. Results from an automated analysis performed by clever software will be returned to the OIC for review. Whatever they make of it will go before the court" (Collie, 2018 p154).

In the context of peer review difficulties, the use of unregulated private firms may seek to decrease forensic science work standards (Donnelly and Scott, 2012). Whilst overarching regulation of the DF industry is an issue, transparency in private DF firms may also be a concern. Whilst tendered processes may force the revelation of internal processes for quality management, behind closed doors, the enforcement of these is solely down to the organisation themselves. Further, although generic governance of ISO 17025 may define a consistent standard to be achieved, once validated under assessment, they may not fully uphold the QM processes which met this standard during subsequent periods of operation. The problem is, there is no real way to tell if this is the case given a lack of consistent quality assessments of the work of DF organisations. At present there are few methods in place to hold organisations accountable to such standards and emphasis must be placed upon each individual engaged in forensic processes to uphold standards. This is alluded to by Tully (2017b).

It is imperative that all staff working in forensic science understand the importance and impact of their work in the criminal justice system (CJS), the importance of quality standards and the provision of robust science. I expect senior management in all organisations to champion the fact that quality is not a conflicting priority with delivery. They should also foster a culture where staff know how to escalate improvement ideas as well as reporting near misses, non-conformities and challenging actions or decisions that detract from quality or good science (Tully, 2017b).

\subsection{What lessons can be learned?}


DF has not yet developed comparable QM procedures as seen in other forensic science disciplines and achieving this is not straightforward. Fundamentally, differing evidence types provide different challenges when assessing quality and unlike traditional forensic sciences, the diversity of digital evidence types means any developed review procedures are likely to be burdensome for those involved if a reliable appraisal of the accuracy of any reported digital evidence is to be established. To draw focus on this work, attention is placed upon the quality management systems available to provide assurance that the work provided by DF practitioner is robust. When distilled, peer review of DF work has, and continues to be, an issue. Where work within other forensic disciplines is verified or checked by separate parties, in DF, typically a practitioner is responsible for their own caseload and this includes the investigation of any exhibits. Checking of and validation of findings remains minimal.

A consensus may be present that 'fact checking' and verification of work may be a job for the defence, where practitioners acting for the prosecution simply propose potentially viable scenarios which a defence must refute and identify errors. This situation is flawed. First, it assumes that those operating in a defence capacity are competent to do so. Whilst many practitioners may be, procedural elements can interfere with the process of ensuring a full investigation of prosecution findings can take place (for example, failure to provide access to original exhibits, images etc). Second, it assumes that the use of a defence can be afforded or is in place. This is becoming a greater issue due to concerns over defence work payment, where less practitioners are engaging in this type of work. Finally, this approach also assumes that it is acceptable for errors to exist and be acknowledged by the defence. In reality this is a significant risk as both prosecution and defence should employ measures which rigorously examine the quality of their work in order to identify errors, preventing potential miscarriages of justice.

If it is assumed that re-examination and verification reviews offers a non-viable method for many organisations to check the standard of their work, alternative considerations should be evaluated.

Dual-investigator. The ACPO (n.d.) Good Practice and Advice Guide for Managers of eCrime Investigation, advises the use of dual tool verification of results to improve reliability. Such advice assumes that any potential issues lie with the validity of a tool in question as opposed to the practitioner. However, the issue remains that whilst both tools may provide the same result (as one would hope to expect), dual-tooling is unlikely to prevent misinterpretation of evidence. The concept of 'dual-examining' is offered for consideration. Whilst not a wholesale solution, dual-examining requires each DF investigation to be divided amongst multiple practitioners responsible for different elements of the work. This has the impact of assigning dual-responsibility, thus encouraging review and collaboration at all stages of an investigation. Further, the division of tasks will naturally foster a relationship of peer review as both practitioners seek to report upon the evidential findings within their work. Dual-examining does not double to workload like a re-examination, and add the benefit of multiple practitioners being able to collaborate during an investigation, exposing findings to twice the scrutiny and twice the discipline specific experience and investigatory knowledge. In essence, this approach relies on the concept that the more people involved, the greater the chance of investigatory errors being avoided or identified. 
Random/Dip-sampling of cases: Dip-sampling is utilised within the examination of fingerprint for quality control purposes (see Section 3.2). Such measures are arguably flawed from the outside as fundamentally, they look to discover constantly applied bad practice which may have been occurring for prolonged periods of time. Whilst this may be present in DF case work, the diversity of work and exhibit types means that this is less likely to be apparent in any sampling-based process. A comparative example is required. For example, the methods for the examination of blood may develop, yet the product, blood, has arguably not. In comparison, concurrent procedures to forensically examine mobile phones have developed alongside the handsets themselves. As a result, whereas the examination for blood has typically three techniques for example (KM, LMG and Hemastix $\mathbb{R}$ ), DF $m$ ay have multiple techniques to examine thousands of different devices types. The result of this means an almost infinite number of variables are in need of checking, an arguably insurmountable task within the confines of a peer review.

What is key to note is that despite being the youngest forensic science discipline, and having the experiences of other areas to learn from, DF operates with arguably the least robust QM procedures of all of the forensic sciences. This should be a concern to those relying on forms of digital evidence as part of sound legal decision making as part of criminal procedures, and measures to support the development of QMs are needed. Given that this task creates significant difficulty, a collaborative effort is required by those involved in digital investigations in order to determine a suitable procedure for achieving high quality work.

\section{Conclusion}

This work has provided an examination of the quality assurance measures in place across the forensic science disciplines of DNA, body fluids and fingerprint examination. Drawing on more 'tried and tested' quality assurance procedures and the external regulatory environment, suggestions are provided for incorporation into the field of digital forensics. It is advised that providers of digital forensic examinations seek to integrate additional quality measures in order to reassure the Criminal Justice System that any results presented are valid and consistent, meeting the standards required of ISO accreditation and those laid out within the Codes of Practice and Conduct.

\section{References}

ACPO (n.d.) 'ACPO Managers Guide: Good Practice and Advice Guide for Managers of eCrime Investigation' Available at: http://www.digital-detective.net/digital-forensicsdocuments/ACPO Good Practice and Advice for Manager of e-Crime-Investigation.pdf (Accessed 17 May 2018)

Agarwal, A., Gupta, M., Gupta, S. and Gupta, S.C., 2011. Systematic digital forensic investigation model. International Journal of Computer Science and Security (IJCSS), 5(1), pp.118-131.

BBC News (2012) 'DNA test jailed innocent man for murder' Available at: http://www.bbc.co.uk/news/science-environment-19412819 (Accessed 17 May 2018)

BBC News (2015a) 'Warning by spending watchdog over forensic science work' Available at: http://www.bbc.co.uk/news/science-environment-30909722 (Accessed 17 May 2018) 
BBC News (2015b) 'Can we still rely on DNA sampling to crack crime?' Available at: http://www.bbc.co.uk/news/uk-32546183 (Accessed 17 May 2018)

BBC News (2016) 'Forensic science strategy vague and incoherent, MPs say' Available at: http://www.bbc.co.uk/news/uk-37390923 (Accessed 17 May 2018)

BBC News (2017a) 'Forensic science standards 'at significant risk" Available at: http://www.bbc.co.uk/news/uk-38527830 (Accessed 17 May 2018)

BBC News (2017b) 'Randox forensics inquiry: police suspend drug-test contracts'. Available at: https://www.bbc.co.uk/news/uk-england-manchester-42144231 (Accessed 21 June 2018)

Bowcott, Owen (2018) 'Solicitor for student in rape case criticises police and CPS' Available at: https://www.theguardian.com/uk-news/2018/jan/30/met-police-and-cps-apologise-to-manafter-collapse-of-case (Accessed 17 June 2018)

BSI Standards Limited (2014) ISO Guide 80: 2015 (E). Guidance for the in-house preparation of quality control materials (QCMs).

BSI Standards Limited (2015a) BE EN ISO 9000: 2015. Quality management systems: Fundamentals and vocabulary.

BSI Standards Limited (2015b) BS EN ISO 9001: 2015. Quality management systems: Requirements.

BSI Standards Limited (2018) BS EN ISO 17015: 2017. General requirements for the competence of testing and calibration laboratories.

Christensen, A. M., Crowder, C. M., Ousley, S. D. and Houck, M. M. (2014), Error and its Meaning in Forensic Science. J Forensic Sci, 59: 123-126. doi:10.1111/1556-4029.12275

Clarke, Lord Justice (2001) Public inquiry into the identification of victims following major transport accidents. Available at:

http://www.integer.co.uk/resources/Clarke\%20Report 2001.pdf Accessed 21 June 2018

Cohen, F.B., 2012. Digital forensic evidence examination. Fred Cohen \& Associates.

Collie, J., 2018. Digital Forensic Evidence - Flaws in the Criminal Justice System. Forensic Science International. 289, Pp 154-155.

CPS (2017) 'Streamlined Forensic Reporting Guidance and Toolkit' Available at:https://www.cps.gov.uk/legal-guidance/streamlined-forensic-reporting-guidance-and-toolkit Accessed 11th June 2018

Crown Court for Northern Ireland Decisions, (2007) The Queen -V- Sean Hoey. http://www.bailii.org/nie/cases/NICC/2007/49.html Accessed 11th June 2018

Donnelly, D. and Scott, K. eds., 2012. Policing Scotland. Routledge. 
Fingerprint Inquiry, The (2011) The Fingerprint Inquiry Report.

https://www.webarchive.org.uk/wayback/archive/20150428160106/http:/www.thefingerprintin quiryscotland.org.uk/inquiry/files/TheFingerprintInquiryReport High res.pdf Accessed 11th June 2018.

Forensic Science Regulator (2016) Guidance: The control and avoidance of contamination in crime scene examination involving DNA evidence recovery. Issue 1.

https://assets.publishing.service.gov.uk/government/uploads/system/uploads/attachment da ta/file/536827/FSR-anti-contamination.pdf (Access 12 June 2018)

Forensic Science Regulator (2017a) Codes of Practice and Conduct for forensic science providers and practitioners in the Criminal Justice System. Issue 4.

https://www.gov.uk/government/uploads/system/uploads/attachment data/file/651966/100 20171009 - The Codes of Practice and Conduct -

Issue 4 final web web pdf 2 .pdf Accessed 14th Mar 2018

Forensic Science Regulator (2017b) Codes of Practice and Conduct. Fingerprint

Comparison, FSR-C-128. Issue 2.

https://www.gov.uk/government/uploads/system/uploads/attachment data/file/638254/128 F

SR fingerprint appendix Issue2.pdf Accessed 14th Mar 2018

Higher Education Academy (2018) Surface learning. Available at:

https://www.heacademy.ac.uk/knowledge-hub/surface-learning (Accessed 11 July 2018)

Ireland, J. and Beaumont, J., 2015. Admitting scientific expert evidence in the UK: reliability challenges and the need for revised criteria-proposing an Abridged Daubert. Journal of Forensic Practice, 17(1), pp.3-12.

Justice 1 Committee Report, (2007) Inquiry into the Scottish Criminal Record Office and Scottish Fingerprint Service. Volume 1: Report.

http://archive.scottish.parliament.uk/business/committees/justice1/reports-07/j1r07-03-vol100.htm Access 11th June 2018.

Justice Committee (2018) Oral evidence: Disclosure of evidence in criminal cases, HC 859

Kind, S. (1957) The use of the Acid Phosphatase test in searching for seminal stains. Journal of Criminal Law and Criminology, 47 (5) Pp 597-600.

Kleuskens, Ralph (2015) 'Forensic Science Quality Management: Errors and Incidents' Available

at:

https://www.nist.gov/sites/default/files/documents/2016/11/22/forensic quality management errors incidents.kleuskens.plenary.pdf

Köhn, M., Olivier, M.S. and Eloff, J.H., 2006, July. Framework for a Digital Forensic Investigation. In ISSA (pp. 1-7). 
Langenburg, G., Champod, C. and Wetheim, P. (2009) Testing for potential contextual bias effects during the verification stage of the ACE- $V$ methodology when conducting fingerprint comparisons. Journal of Forensic Sciences, 54 (3) Pp 571 - 582

Ludwig, A. and Fraser, J., 2014. Effective use of forensic science in volume crime investigations: Identifying recurring themes in the literature. Science and Justice, 54(1), pp.81-88.

Morgan, R.M., 2017. Conceptualising forensic science and forensic reconstruction. Part I: A conceptual model. Science \& Justice, 57(6), pp.455-459.

Murphy, E., 2007. The new forensics: Criminal justice, false certainty, and the second generation of scientific evidence. Cal. L. Rev., 95, p.721.

Meyers, M. and Rogers, M., 2004. Computer forensics: The need for standardization and certification. International Journal of Digital Evidence, 3(2), pp.1-11.

National Academy of Sciences (2009) Strengthening Forensic Science in the United States: A Path Forward. Committee on Identifying the Needs of the Forensic Sciences Community. Available at: http://www.nap.edu/catalog/12589.html Accessed 16th Feb 2018

National Police Chiefs' Council (2018) History and Background. Available at: http://www.npcc.police.uk/About/History.aspx Accessed on 16th Feb 2018

Osborne, N.K.P. and Taylor, M.C., (2018) Contextual information management: An example of independent-checking in the review of a laboratory-based bloodstain pattern analysis.

Science \& Justice, 58. Pp 226 - 231.

Parliament.tv (2018) 'Justice Committee' Available at: https://www.parliamentlive.tv/Event/Index/13d15d6a-8aa9-40ce-bdf2-3d19777b3af8 (Accessed 16 May 2018)

Pollitt, Mark. Casey, Eoghan. Jaquet-Chiffelle, David-Olivier and Gladyshev, Pavel (2018) 'A Framework for Harmonizing Forensic Science Practices and Digital/Multimedia Evidence' Available at:

https://www.nist.gov/sites/default/files/documents/2018/01/10/osac ts 0002.pdf (Accessed 16 May 2018)

Rawley, A. and Caddy, B. (2007) Damilola Taylor: An independent review of forensic examination of evidence by the Forensic Science Service. Available at: http://netk.net.au/Damilola/Damilola2007.pdf (Accessed 11 July 2018)

Selamat, S.R., Yusof, R. and Sahib, S., 2008. Mapping process of digital forensic investigation framework. International Journal of Computer Science and Network Security, 8(10), pp.163-169.

Smith, Andrew (2018) 'Failed by Forensics' Available at: https://www.bbc.co.uk/programmes/b0b5t824 (Accessed 4 July 2018) 
SWGFAST, (2013) Document \#10. Standards for Examining Friction Ridge Impressions and Resulting Conclusions (Latent/Tenprint). Version 2.0.

http://clpex.com/swgfast/Documents.html (Accessed 1 June 2018)

Smit, N.M., Morgan, R.M. and Lagnado, D.A., 2017. A systematic analysis of misleading evidence in unsafe rulings in England and Wales. Science \& Justice.

Skelly, B. (2018) 'NPCC responds to ONS crime statistics' Available at:

https://news.npcc.police.uk/releases/npcc-lead-for-crime-recording-statistics-responds-toons-crime-statistics (Accessed 1 June 2018)

Skills for Justice (2013) SFJ CN702: Determine forensic examinations to be undertaken for exhibits. https://www.ukstandards.org.uk/PublishedNos/SFJCN702.pdf (Accessed 12 June 2018)

Sommer, P., 2018. Accrediting digital forensics: What are the choices?. Digital Investigation 25 (2018) $116 \mathrm{e} 120$

Sloan, J. (2015) 'There's no code of ethics to govern digital forensics - and we need one' Available at: https://theconversation.com/theres-no-code-of-ethics-to-govern-digitalforensics-and-we-need-one-45755 (Accessed 1 June 2018)

Teesside University (2008) Programme Specification: Fd Sc Forensic Practice (Fingerprint Identification). Available at:

https://www.tees.ac.uk/docs/DocRepo/Programme\%20specifications/School\%20of\%20Scien ce\%20and\%20Engineering/FdSc\%20Forensic\%20Practice\%20(Fingerprint\%20Identification L.doc (Access 11 July 2018)

Tully, G. (2017) 'Annual Report' Available at:

https://assets.publishing.service.gov.uk/government/uploads/system/uploads/attachment da ta/file/674761/FSRAnnual Report 2017 v1 01.pdf (Accessed 1 June 2018)

Tully, G. (2017b) 'Message from the Regulator' Available at: https://assets.publishing.service.gov.uk/government/uploads/system/uploads/attachment da ta/file/648320/FSR Newsletter 29.pdf (Accessed 1 June 2018)

Vincze, E.A., 2016. Challenges in digital forensics. Police Practice and Research, 17(2), pp.183-194.

Walvisch, J. (2017) 'Fingerprinting to solve crimes: not as robust as you think' Available at: http://theconversation.com/fingerprinting-to-solve-crimes-not-as-robust-as-you-think-85534 (Accessed 1 June 2018)

White, R.M. and Greenwood, J.J., (1988). DNA Fingerprinting and the Law. The Modern Law Review, 51(2), pp.145-155. 
Wilson, C. (2011) 'Digital Evidence Discrepancies - Casey Anthony Trial' Available at: http://www.digital-detective.net/digital-evidence-discrepancies-casey-anthony-trial/ (Accessed 1 June 2018) 